\title{
On the microscopic mechanism behind the purely orientational disorder-disorder transition in the plastic phase of 1 -Chloroadamantane
}

\author{
A. Vispa, ${ }^{1}$ D. Monserrat, ${ }^{1}$ G. J. Cuello, ${ }^{2}$ F. Fernandez-Alonso,${ }^{3,4}$ S. \\ Mukhopadhyay, ${ }^{3,5}$ F. Demmel, ${ }^{3}$ J.Ll. Tamarit, ${ }^{1}$ and L. C. Pardo ${ }^{1}$ \\ ${ }^{1}$ Grup de Caracterització de Materials, Departament de Física, EEBE, \\ Universitat Politècnica de Catalunya, Eduard Maristany 10, E-08019 Barcelona, Catalonia, Spain \\ ${ }^{2}$ Institut Laue Langevin, 6, rue Jules Horowitz (38042), Grenoble, France \\ ${ }^{3}$ ISIS Facility, Rutherford Appleton Laboratory, Chilton, Didcot, Oxfordshire OX11 0QX, UK \\ ${ }^{4}$ Department of Physics and Astronomy, University College London, Gower Street, London, WC1E 6BT, UK \\ ${ }^{5}$ Department of Materials, Imperial College London, \\ Exhibition Road, London SW7 2AZ, United Kingdom
}

(Dated: May 30, 2017)

\begin{abstract}
Globular molecules of 1-Chloroadamantane form a plastic phase in which the molecules rotate in a restrained way, but being their centers of mass forming a crystalline ordered lattice. Plastic phases can be regarded as test cases for the study of disordered phases since, contrary to what happens in the liquid phase, there is a lack of stochastic translational degrees of freedom. When the temperature is increased a hump in the specific heat is observed indicating a change in the energetic footprint of the dynamics of the molecules. This change takes place without a change in the symmetry of the crystalline lattice, i.e. no first-order transition is observed between temperatures below and above the calorimetric hump. This implies that subtle changes in the dynamics of the disordered plastic phase concerning purely orientational degrees of freedom should appear at the thermodynamic anomaly. Accordingly, we describe, for the first time, the microscopic mechanisms behind a disorder-disorder transition through the analysis of neutron diffraction and QENS experiments. Results evince a change at the molecular rotational dynamics accompanied by a continuous change in density.
\end{abstract}

PACS numbers: 33.15.Fm,61.20.Ja,61.20.-p,61.25.-f

Molecular disordered systems, such as liquid and plastic phases, are still far to be completely understood. Even simple systems with very restricted disorder, as those where atoms have a fractional occupancy of crystallographic sites, are still a matter of controversy [1-4]. In order to gain some insights about the role of disorder into the properties of matter, the study of systems with constrained disorder is very relevant. In the case of our work, we have studied a plastic phase for which only the orientational disorder is relevant, being the positional order restrained since the molecular centers-ofmass occupy a well-defined lattice position. This fact makes these systems very interesting models for canonical liquids. Specifically, they are useful to investigate the role of translational and orientational degrees of freedom in disordered phases. For liquids this is accounted in the so-called two-order parameter description of liquids [5-11] that try to give an explanation of the liquidliquid transition phenomenon. In that case, the change of molecular ordering when passing through the liquidliquid transition is known to locally change the relative position of the molecular mass centers, and is associated with a transition from a low-density liquid to a high-density ordinary liquid. No change in the relative orientation of the molecules has been reported between the two liquid states. Such a liquid-liquid phase transition is one of the main puzzling hypothesis used to explain the properties of water [12].

In a previous study,[13, 14] we showed that liquid trans-dichloroethylene exhibits a continuous change in both dynamics and structure when studied as a function of temperature, i.e. there is a change form a high density to a low density liquid without the occurrence of a first order phase transition. The primary aim of the present work is to assess whether a similar phenomenology applies to plastic-crystalline phases by studying the anomalous thermal anomaly presented 1 -Chloroadamantane as a test case.

On the other hand, the liquid-liquid transformation is associated with a first-order phase transition that is associated with a discontinuity in the density of the liquid (and any other physical value describing the phases). This has currently been explained by the change in the local molecular arrangement of the centers of mass of the molecules [15, 16]: it implies, thus a change in translational order. The second goal of our work is to determine if it is possible the existence of a purely orientational disorder-disorder phase transition without noticeable change in the molecular position, i.e. a change purely driven by the reorientational dynamics. To do that we will study the plastic phase of 1-Chloroadamantane whose crystalline structure is well known. This has the advantage that we can easily separate the effects of changes in relative position and orientation of the molecules, since any change in molecular packing due to a different relative molecular position will be reflected in a change of the Bragg peaks. The study of a plastic phase allows us thus to unambiguously dif- 
ferentiate changes in the translational and orientational molecular arrangements in a disorder-disorder transition.

1-Chloroadamantane $\left(\mathrm{C}_{10} \mathrm{H}_{15} \mathrm{Cl}\right)$, hereafter $\mathrm{ClA}$ and shown in Fig.e 1, is a rigid spherical-like molecule with $C_{3 v}(3 m)$ point-group symmetry. It is obtained from the cage-like molecule adamantane $\left(C_{10} H_{16}, T_{d}\right.$ symmetry $)$ via the substitution of an aliphatic hydrogen at a tertiary carbon by chlorine. When heating the low-temperature crystalline phase, ClA displays a first-order solid-solid phase transition at $248.65 \mathrm{~K}$ [17] between the monoclinic $P 2_{1} / c$ ordered phase and the $F m 3 m$ plastic phase [1820]. Kobashi et al. performed a series of calorimetric experiments in the stability domain of the plastic phase: a broad heat capacity effect peaking at a temperature of circa $T \approx 320 \mathrm{~K}[17]$. Considering the size of the abnormal heat capacity and the orientational disorder of the plastic phase, this jump does not seem to be related to a Schottky anomaly, as suggested by Kobashi et al., but rather to a change in the orientational arrangement and/or in its underlying dynamics. ClA, together with some other adamantane derivatives, has been the subject of several studies by means of broadband dielectric spectroscopy (BDS) [3, 21-24], nuclear magnetic resonance (NMR) [25, 26], differential thermal analysis (DTA) [24, 27], x-ray scattering [19, 28-31], IR experiments [32], calorimetry [17], quasielastic neutron scattering (QENS) [33, 34] and molecular dynamics (MD) simulations $[35,36]$.

For what concerns the microscopic dynamics, the studies of Bée et al. [34] stated that the motion of $\mathrm{ClA}$ at the lowest temperatures of the plastic phase cannot be explained by a model involving isotropic rotations. Instead, molecular motions were described via recourse to a dynamic structure factor that includes the sum of uniaxial rotations plus jumps between [111] lattice directions, similar to those in 2-adamantanone [3,33]. The model in that work describes the data by using a "rough approximation" (as stated by the authors) consisting of a complete decoupling between the two motions, uniaxial rotation and tumbling.

None of the aforementioned works provided a microscopic explanation of the calorimetric anomaly within the plastic phase of ClA. The only exception are the works of Affouard et al. where an indication of a change in the dynamics at a temperature close to the specific-heat anomaly at $320 \mathrm{~K}$ was found via the concurrent use of MD, NMR, and Raman scattering [35, 36]. However, only a vague explanation was found in those works that ascribed the calorimetric hum to a "local orientational ordering phenomena". The goal of our work is to find the microscopic mechanism that causes a maximum in the specific heat in that temperature range by the concurrent analysis of the changes in both structure and dynamics of ClA within the plastic phase. To achieve this task, we have used both neutron diffraction (ND) and Quasi Elastic Neutron Scattering (QENS). Moreover, we have also investigated whether it is possible to gain some insight about the dynamics of ClA by using a non-isotropic rotation model originally developed to describe polarizationresolved fluorescence data [37]. To the best of our knowledge, this model has not been used before for the analysis of QENS data.

It is of utter importance to investigate the microscopic changes in the studied system since this would suggest that slight modifications of relative molecular orientation may lead to changes into the macroscopic properties (such as density or rotational diffusion) without a change of the structural molecular packing and thus, without the emergence of a first-order phase transition.

The paper is organized as follows: Details of the experimental devices and data analysis are given in Sec. II. Results obtained for neutron diffraction (part A) and quasielastic neutron scattering (part B) are described in Sec. III. Section IV is devoted to the discussion of the results by considering all the available experimental information. Finally, conclusions are gathered in the last section, Sec. V.

\section{EXPERIMENTAL}

\section{A. Sample and Methods}

Deuterated ClA for the neutron diffraction experiments was purchased from CDN Isotopes Inc. with a degree of deuteration of $98 \%$, whereas the hydrogenated compound used for QENS was purchased from Sigma Aldrich Chemical Co. with a purity grade of $98 \%$ and no further purification was performed.

Diffraction measurements were performed using the two-axis powder diffractometer, D1B, at the Institut Laue-Langevin (ILL) in Grenoble (France). The wavelength used to perform the experiment was $\lambda=2.52 \AA$ at the sample position [38]. The sample was contained in a vanadium cylindrical can, that does not contribute to the coherent part of the diffraction pattern. The temperature of the sample was controlled by a cryo-furnace and neutron diffraction measurements were performed between $220 \mathrm{~K}$ and $380 \mathrm{~K}$ using a scanning rate of $0.2 \mathrm{~K} \mathrm{~min}^{-1}$.

QENS experiments were carried out on IRIS time-offlight spectrometer at the ISIS pulsed neutron source at the Rutherford Appleton Laboratory (UK) [39, 40]. To minimize the effects of multiple scattering and achieve a transmission of $\approx 90 \%$, a ClA sample of $0.25 \mathrm{~mm}$ of thickness was kept in a flat aluminum container composed by two slabs with a thickness of $0.1 \mathrm{~mm}$. Sample holder was put in a closed-cycle cryostat at $45^{\circ}$ relative to the incident beam to collect data at $T=$ $260,280,300,320,340,360 \mathrm{~K}$. The instrument is a timeof-flight inverted-geometry spectrometer, so the final en- 


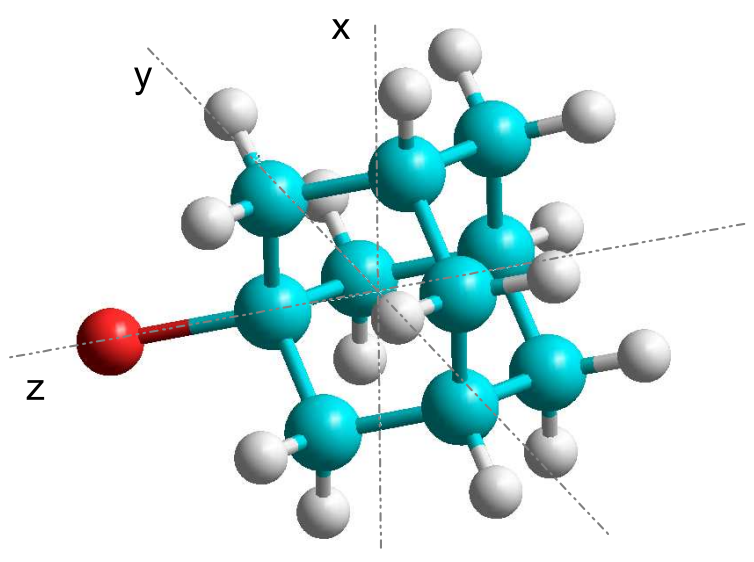

FIG. 1: (Color online) Structure of an ClA: carbon (blue), hydrogens (white) and chlorine (red). Dashed lines depict the three axes of rotation used throughout this work. The $z$ direction has been made to coincide with the $C_{3 v}$ symmetry axis along the carbon-chlorine bond.

ergy has been selected, $E_{\mathrm{f}}=1.84 \mathrm{meV}$, with an energy resolution of $17.5 \mu \mathrm{eV}$. The analyzer bank used the reflection [002] of pyrolitic graphite to record 51 neutron spectra simultaneously, corresponding to a scattering angle $(2 \theta)$ range between $25^{\circ}-160^{\circ}$ and then to a $Q$-range from 0.442 to $1.854 \AA^{-1}$. Data reduction, including empty-cell subtraction, calibration with a vanadium rod, and conversion into constant $Q$ spectra, was performed with the program MANTID [41, 42]. Calculating the momentum transfer $Q$ from the scattering angle, spectra were then grouped together in bins of $0.1 \AA^{-1}$.

The structure factor maximum appears at $\approx 1.15 \AA^{-1}$ [43], therefore, being interested in the study of the incoherent scattering related to the molecular dynamics, the spectra were analyzed in the $Q$-range $0.45-1.05 \AA^{-1}$, where the coherent contribution can be neglected. The energy transfer used to perform data analysis was $|E|<$ $0.5 \mathrm{meV}$

\section{B. Data Analysis}

Neutron Diffraction. Diffraction patterns were analyzed using the FullProf Suite package [44, 45]. The background was taken into account by a fit to the diffuse scattering part of the diffraction data. Pattern matching refinement was applied to the patterns collected as a function of temperature for the face centered cubic (fcc) plastic phase.

QENS. The analysis of QENS data has been performed using a Bayesian ansatz through an adaptive Markov-Chain Monte Carlo (MCMC) algorithm. The main advantages of using a Bayesian algorithm with re- spect to a Frequentist approach are the following: (a) it enables the fitting process not to get stuck in one of the local minima of the parameter hypersurface; (b) it takes into account all possible correlations between the parameters; (c) it is possible to describe the results through Probability Distribution Functions (PDFs) without any underlying assumptions; (d) model selection is performed by using all the available information obtained from the model and the data. All these features are implemented in the software package FABADA developed by our group [46-51] and recently included in the MANTID analysis package $[41,42,51]$.

Bayesian inference performed with this method is done in two parts. First of all, the fitting leads to the set of parameters that better describes the data. In a second step, the software generates a complete Markov chain containing all the parameters that can describe the data, and their associated $\chi^{2}$ merit function. The adaptive algorithm and the Bayesian methodology have been described in detail in previous publications [13, 46-53], and the reader is referred to these works for further details.

In order to avoid the overinterpretation of QENS data on ClA, we have first determined the optimal value of dynamics processes that can be described by data, basically restricted by the errors and the finite range of energy and momentum transfers. To this end, we have used a simple model consisting on a superposition of Lorentzian curves (L1, L2, L3). The scattering law $S(Q, E)$ can be then described as a function of momentum $(\hbar Q)$ and energy $(E=\hbar \omega)$ transfer by:

$$
S(Q, E)=A_{0} \delta(E)+\sum_{i=1}^{n} A_{i} \frac{1}{\pi} \frac{\Gamma_{i}}{E^{2}+\Gamma_{i}^{2}},
$$

where $\Gamma_{i}$ is the width of the i-th Lorentzian function and $A_{i}$ is its corresponding intensity.

The tested physical models aimed to describe the microscopic dynamics of ClA were two: an isotropic rotation and an anisotropic rotation. In the first case a complete free rotation is assumed for the molecule, while the second assumes that $\mathrm{ClA}$ is rotating in a constrained way. It is important to note that this second model does not assume that the rotation around each molecular principal axis is decoupled. Our model assumes an intrinsic asymmetry in the rotation of the molecule instead, and, as mentioned in the introduction, we are not aware of its use to describe QENS data before.

The first model reads:

$$
\begin{gathered}
S_{\text {inc }}^{\text {iso }}(Q, E)=A_{0} \delta(E)+\sum_{l=1}^{\infty} A_{l} \frac{l(l+1) D_{R}}{(E)^{2}+\left[l(l+1) D_{R}\right]^{2}} \\
\text { with } A_{l}(Q)=(2 l+1) j_{l}^{2}(Q r),
\end{gathered}
$$

where $D_{R}$ is the isotropic rotational diffusion parameter and $j_{l}(Q r)$ are spherical Bessel functions of $l$-th order. 
The isotropic rotational model assumes that all the principal axes of inertia for molecular rotation are identical.

The second model used is the anisotropic model of [37], originally developed to describe polarizationsensitive single-molecule fluorescence microscopy. The corresponding scattering law reads:

$$
\begin{aligned}
S_{\text {inc }}^{\text {anis }}(Q, E) & =A_{0} \delta(E)+\sum_{l=1}^{\infty} A_{l} \cdot \sum_{m=-l}^{l} \frac{\Gamma_{l}^{m}}{E^{2}+\left(\Gamma_{l}^{m}\right)^{2}} \\
\text { with } \Gamma_{l}^{m} & =l(l+1) D_{x}+m^{2}\left(D_{z}-D_{x}\right),
\end{aligned}
$$

where $D_{z}$ is the uniaxial rotational diffusion coefficient along the molecular three-fold symmetry axis, and $D_{x}$ is the rotation around the perpendicular axes (see Figure 1 ) and $l$ and $m$ are related to the elements of the Wigner rotation matrix needed to compute the scattering law. The use of this model is justified by the values obtained for the moments of inertia around each of the principal axes since $I_{x} \approx I_{y}\left(I_{z}=295\right.$ a.u. $\cdot \AA^{2}, I_{x}=591$ a.u. $\cdot \AA^{2}$ and $I_{y}=589$ a.u. $\cdot \AA^{2}$, being a.u. atomic units).

The scattering laws of these two models are calculated as two infinite series of terms. If expanded up to the second-order term, they read:

$$
\begin{aligned}
S_{\text {inc }}^{\text {iso }}(Q, E)=A_{0} \delta(E) & +A_{1} L\left(2 D_{R}\right) \\
& +A_{2} L\left(6 D_{R}\right) \\
& \\
S_{\text {inc }}^{\text {anis }}(Q, E)=A_{0} \delta(E)+ & 2 A_{1} L\left(D_{x}+D_{z}\right) \\
& +A_{2} L\left(2 D_{x}\right) .
\end{aligned}
$$

The physical relevance of this truncation is explained in more detail below.

It is noteworthy to point out that the fits to these physically relevant models have been performed using the whole scattering law, i.e. a single function has been used to fit all $Q$-values, which means that the fit has been performed using the complete two-dimensional function $S_{\text {inc }}(Q, E)$. This procedure is very important since the obtained results are described using a minimum number of parameters thus increasing the robustness of the results with respect to a series of fits to single functions for each $\mathrm{Q}$ value.

Model selection. We shortly recall how model selection is being done when using a Bayesian ansatz, for further information the reader is referred to previous works. When performing model selection using $\chi^{2}$ PDFs three scenarios can be found:

- Two non-overlapping PDFs. This means that the model with lower values of $\chi^{2}$ is preferred, since no combination of parameters of the worst model is able to describe better the data than the best model.

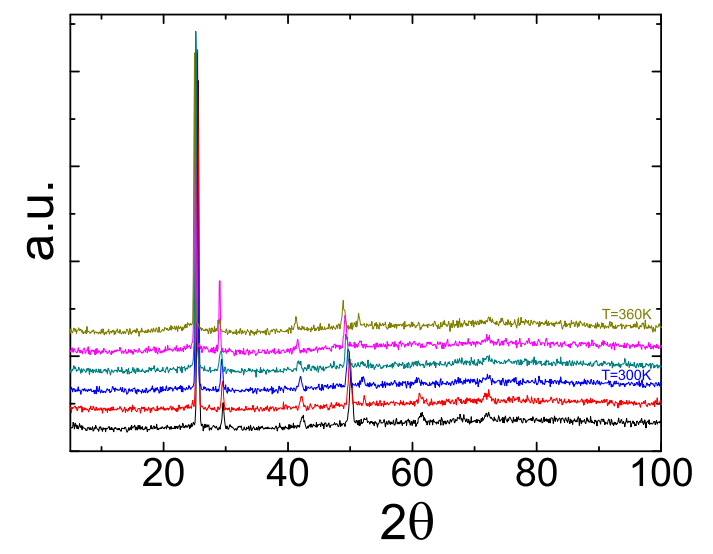

FIG. 2: (Color online) Neutron diffraction patterns obtained for ClA shown every $20 \mathrm{~K}$ starting at $260 \mathrm{~K}$.

- Two partially-overlapping PDFs. In this case the maximum (most-probable value) of the $\chi^{2}$ distribution should be chosen as a criterion to find the best model. However, in this case we should be aware that it is possible that any new piece of information or data can reverse the model-selection process.

- Two completely overlapping PDFs. If the two PDFs are so similar such that no clear difference is found either in the minimum $\chi^{2}$ or in the maximum of the PDF, the model with less parameters should be chosen following Occam's razor[56, 57].

It should be pointed out that the obtained $\chi^{2}$ PDFs will be obtained in this work assuming that we have not a prior preference for any model, i.e., we use a maximum ignorance prior to be as objective as possible. Of course, any new piece of information may change the selection of the better model, specially if the PDFs describing the different models overlap.

\section{RESULTS}

\section{A. Neutron Diffraction}

The neutron diffraction patterns obtained for ClA as a function of temperature can be seen in Fig. 2. It is evident, given the resemblance of the patterns before and after the thermal event in the calorimetric data, that the crystalline structure at low and high temperatures is exactly the same. This confirms that there are no differences in the overall molecular structural packing, i.e. the relative molecular position of the molecular centers of mass, and that the differences between the plastic phase at low and high temperature regions must be of a purely orientational nature. 
The results obtained from the analysis of diffraction measurements show a step-like evolution of the peak position of the [111] Bragg reflection as shown in Fig. 3a. We plot also in panel $b$ of the same figure the lattice parameter of the cubic structure obtained from the fitting of the whole diffraction pattern. As it is clear from this figure, the step-like feature in the structural measurement is observed at the same temperature where the calorimetric hump observed by Kobashi et al. [17] takes place.

Structural measurements thus, show a continuous change within the plastic phase between a high-density region (at low temperature) and a low-density region (at high temperature). This unambiguously demonstrates that the change between the two states is performed without the occurrence of a first-order phase transition. In order to disclose the orientational correlation changes across the continuous density change where the heat capacity anomaly appears, we devote the next section to the study of the rotational dynamics of the system.

\section{B. QENS}

The first question to be answered about the obtained QENS results is the maximum number of distinct relaxation processes compatible with the experimental data. Paraphrasing the seminal work of Sivia et al. we want to know "how many lines are there" $[51,54,55]$. In order to determine the optimal number of Lorentzians that describe our data we have calculated the $\chi^{2}$ PDFs for one, two, and three Lorentzians. A first glance at Figure 4 makes it evident that one Lorentzian is not able to describe the QENS data satisfactorily, as shown in the figure inset. Since two- and three-Lorentzian models give virtually identical $\chi^{2} \mathrm{PDFs}$, the simpler model has been chosen for further analysis, as stated in a previous section.

In order to infer the spatial character of the motions associated with these two processes, the spectral widths $\Gamma_{\mathrm{i}}$ have been analyzed over the entire $Q$-range and at all temperatures. The results for $T=340 \mathrm{~K}$ are shown in Fig. 5. Their insensitivity to $Q$ evidences the presence of localized motions, a result which is consistent with the orientational disorder of the translationally ordered plastic phase.

To unravel the microscopic mechanism of ClA dynamics in the plastic phase, we have performed a Bayesian analysis of the QENS data using the isotropic and anisotropic models explained earlier to describe the dynamic structure factor. Figure 6 shows the fits of both models at high temperatures at a given value of $Q$. Although only an instance is shown in the figure, we would like to point out that that they have been obtained using the entire scattering law. The values obtained for $D_{R}$ $(\approx 0.03 \mathrm{meV}$ at $T=340 \mathrm{~K})$ using the isotropic model

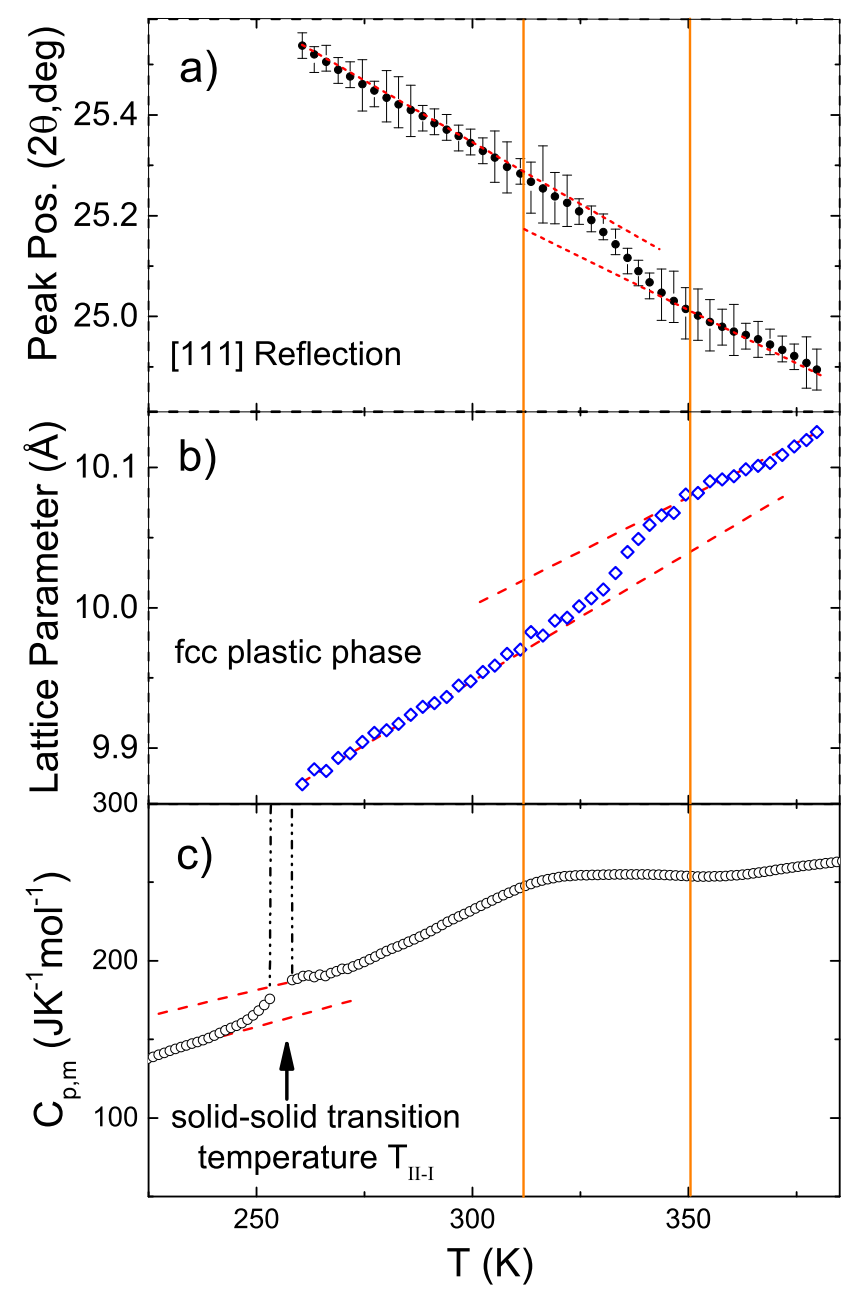

FIG. 3: (color online) Peak position of the [111] reflection (a) and lattice parameter of the cubic cell (b) as a function of temperature.(c) Calorimetric measurements of Kobashi et al. [17] where the specific heat anomaly is to be seen.

are in good agreement with the work of Bée [34] (0.042$0.056 \mathrm{meV}$ at $T=353 \mathrm{~K}$ ).

Visual inspection of the data fits in this figure indicates that the anisotropic model provides a better description of the experimental QENS data. This better agreement is particularly good at high energy transfers, i.e. at the "wings" of the obtained experimental data. In order to perform a quantitative model selection, Fig. 7 reports the corresponding $\chi^{2}$ PDFs: When truncated at the second term, the anisotropic model describes the data much better that the isotropic one, that is, there is no overlap between PDF distributions. Furthermore, even the inclusion of a third term in the isotropic model does not lead to a noticeable improvement of the anisotropic model relative to the anisotropic model. The same considerations apply to all temperatures investigated in this work and, thus, we can conclude that the dynamics of ClA up to 


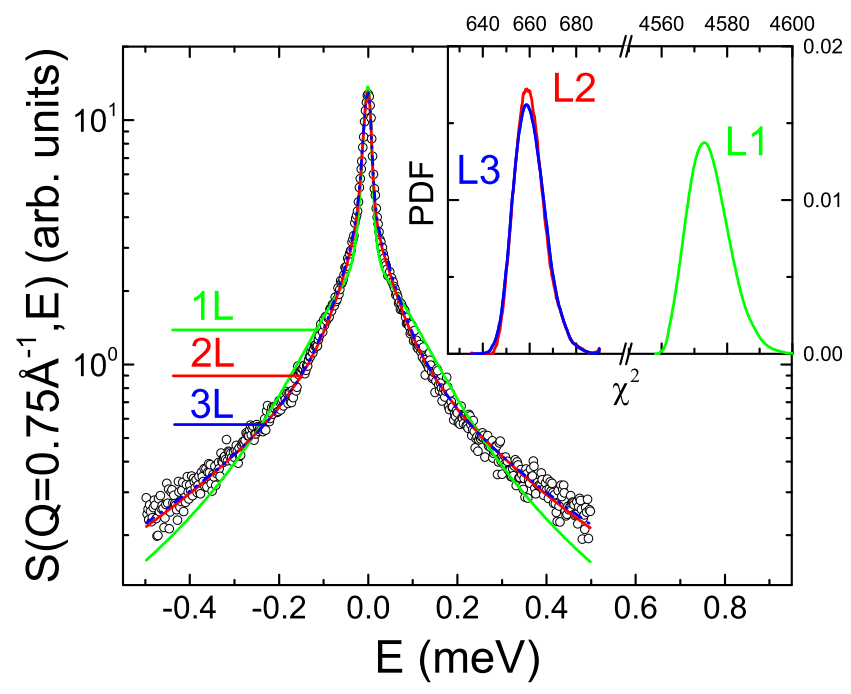

FIG. 4: (color online) Data fits for $\mathrm{ClA}$ at $T=340 \mathrm{~K}$ and $S\left(Q=0.75 \AA^{-1}, E\right)$ using different models: one (L1, green line), two (L2, red line), and three Lorentzians (L3, blue line). Using the same colors, the inset shows the corresponding $\chi^{2}$ PDFs.

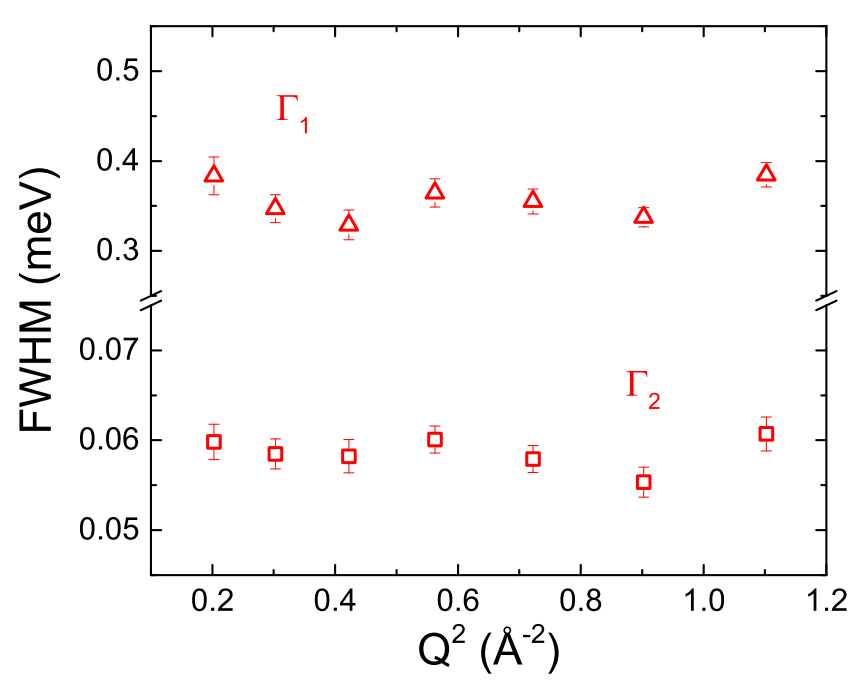

FIG. 5: (color online) Dependence of spectral line widths $\Gamma_{i}$ (FWHM) of $S(Q, E)$ with $Q^{2}$ at $T=340 \mathrm{~K}$ for the two relaxation processes.

$360 \mathrm{~K}$ is anisotropic in nature.

Once the anisotropic model has been selected as the one that describes better the data across the entire temperature range, we can study the physical parameters derived from that model. The rotational diffusion coefficient values $D_{x}$ and $D_{z}$ obtained by the anisotropic model differ about one order of magnitude (Figure 8), describing a much-faster rotation around the $z$-axis relative to the other two orthogonal directions. The dif-

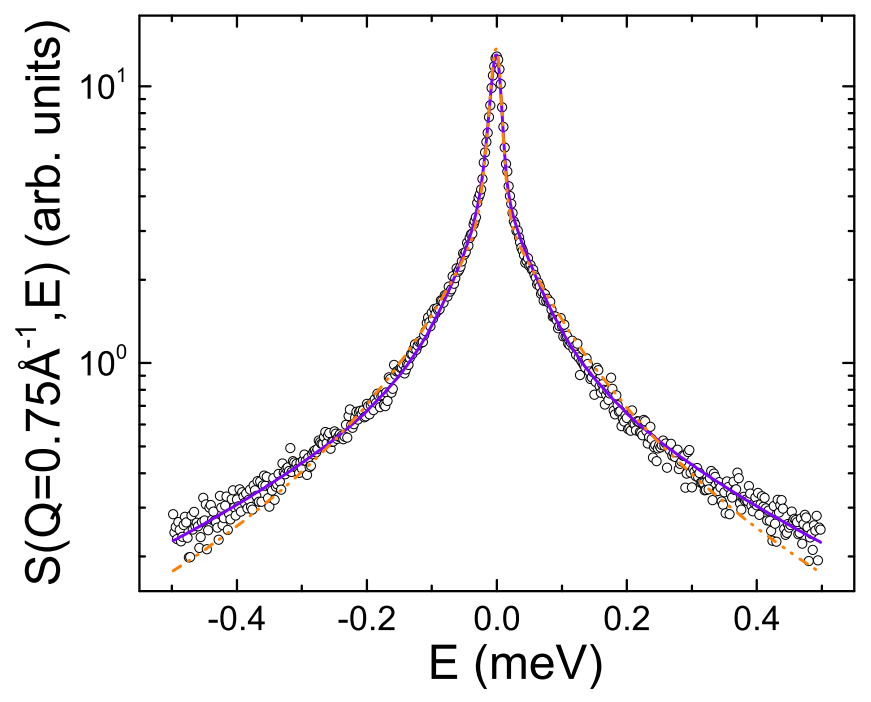

FIG. 6: (color online) Anisotropic (purple line) vs Isotropic (dashed orange line) models for ClA at $T=340 \mathrm{~K}$ and $Q=$ $0.75 \AA$. The models are presented truncated to the secondorder term (see equation 4)

ference between them decreases with increasing temperature, i.e. the dynamics tends to be isotropic, but even at the highest temperature they still differ by about a decade. Rotation around the three-fold molecular symmetry axis z (see Fig. 1) exhibits a mild temperature dependence in the low-temperature region, whereas at higher temperatures this behavior changes leading to an increase of the rotational diffusion coefficient $D_{z}$. This change of the dynamics goes along with a change of slope of $D_{x}$ (see Figure 8) which demonstrates a change in the rotational dynamics at temperatures close to the heat capacity anomaly.

Moreover, such reorientational processes, at least for the well-known 1-X-adamantane derivatives, are mainly dominated by the slower motions corresponding to the overall molecular tumbling, whereas the uniaxial rotations along the dipolar C3 axis are much faster and with a much smaller activation energy. In other words, these facts represent the well-known statement that translation and rotation have no effects on the experimental structure factors of the orientationally disordered phases [58].

\section{DISCUSSION}

Figures 3 and 8 clearly show that there is a continuous change in the structure (lattice constant, i.e. density) and rotational dynamics at the same temperature where the calorimetric hump is observed. Before doing any further analysis, it is important to state that the calorimetric effect, the change in the density and the rotational 


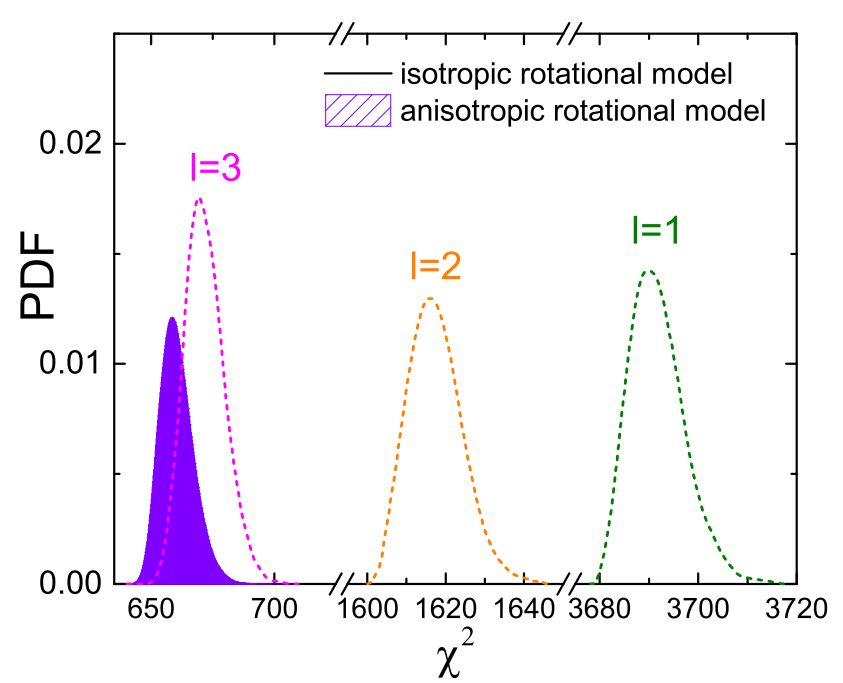

FIG. 7: PDFs obtained from global fits over the entire scattering law. Solid area describes the anisotropic model while dotted lines describe isotropic ones.

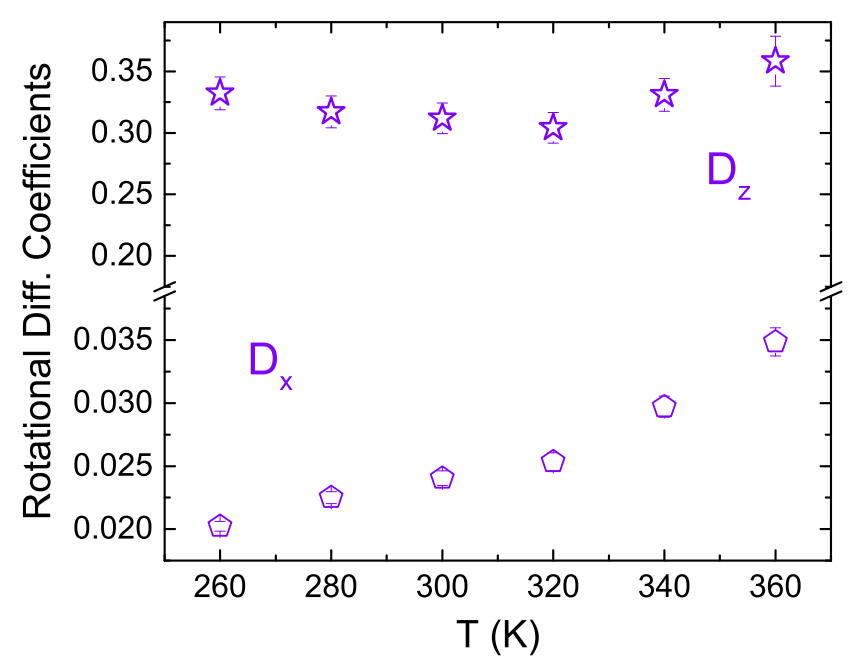

FIG. 8: (color online) Rotational diffusion coefficients as a function of temperature.

dynamics show consistent changes in a continuous way, i.e., there is not a discontinuity in any reported physical magnitude.

Concerning the structure, thermal expansion at both high- and low- temperature sides is similar and, therefore, there is not a significant change in the expansivity of the material in these two regions. However there is a continuous change in the lattice parameter at about $320 \mathrm{~K}$ thus indicating an increase of the free volume needed for the molecules to rotate. On the other hand, figure 8 shows the change in the dynamics of the rotation of the molecule around its symmetry axis $z$, and the rotation associated with the jumps between equivalent orientations of the molecule in the lattice $D_{x}$. Taking into account the momentums of inertia of the isolated molecule, the ratio $D_{z} / D_{x}$ could be calculated as $D_{z} / D_{x}=\sqrt{I_{x} / I_{z}}=1.4$. In order to figure out how far is the dynamics from a free anisotropic rotation only restricted by the molecular shape (and not impeded by interactions with neighbour molecules) we show in Fig. 9 the value for $D_{z} / D_{x}$ as a function of temperature. These values are substantially larger than those expected from the moments of inertia of the isolated molecule. This result strongly supports that the uniaxial motions around the $\mathrm{z}$ and the $\mathrm{x}$ axes of the molecule inside the crystal cannot be explained simply by the molecular shape, otherwise the measured $D_{z} / D_{x}$ should be close to that of the free rotating molecule around its principal axes $\left(D_{z} / D_{x}=1.4\right)$. The only way to explain the huge difference of $D_{z} / D_{x}$ between that of the non-interacting free molecule and that of the real cooperatively interacting molecules in the crystalline disordered phase is that the motions, in the second case, are dominated by the local environment.

The temperature dependence of rotational diffusion coefficients shown in Fig. 8 provides further physical insights into the microscopic behaviour of ClA in the plastic phase. These data show that $D_{z}$ is not only much larger in magnitude than $D_{x}\left(D_{z}>10 \times D_{x}\right)$ but also exhibits a marked insensitivity to temperature. Furthermore, $D_{x}$ increases monotonically up to the melting transition. Based on the anisotropic model for the dynamic structure factor introduced in this work, these differences can be rationalized on the basis of the overall strength of intermolecular interactions underpinning $\mathrm{ClA}$ stochastic rotational motions either parallel $\left(D_{z}\right)$ or perpendicular $\left(D_{x}\right)$ to the $\mathrm{C}-\mathrm{Cl}$ three-fold axis. In the former case, these interactions primarily involve weak couplings between peripheral hydrogens of neighboring $\mathrm{ClA}$ molecules, and these are fully activated as soon as the plastic phase is accessed from the ordered solid. Such a different behaviour of the rotational dynamics has already been observed in much-simpler dumb-bell fluids, including hydrogen fluoride [59-61]. In contrast with the above, rotational motions associated with $D_{x}$ necessarily require the disruption of stronger intermolecular interactions involving the more polarisable $\mathrm{Cl}$ atom and its associated $\mathrm{C}-\mathrm{Cl}$ dipole moment, a situation that necessarily translates into a marked temperature dependence of these dynamical modes. These considerations also provide the starting point to attain a microscopic understanding of the anomaly observed in the heat capacity data shown in Fig. 3, whereby relatively slow $D_{x}$ reorientations are ultimately responsible for the disruption of dipolar order in the plastic phase, an effect which also leads to a subtle (yet measurable) expansion of the underlying ClA lattice well before melting. The calorimetric jump seen can thus be rationalized by the change in cooperativity of the reorientational dynamics in an anisotropic way. 


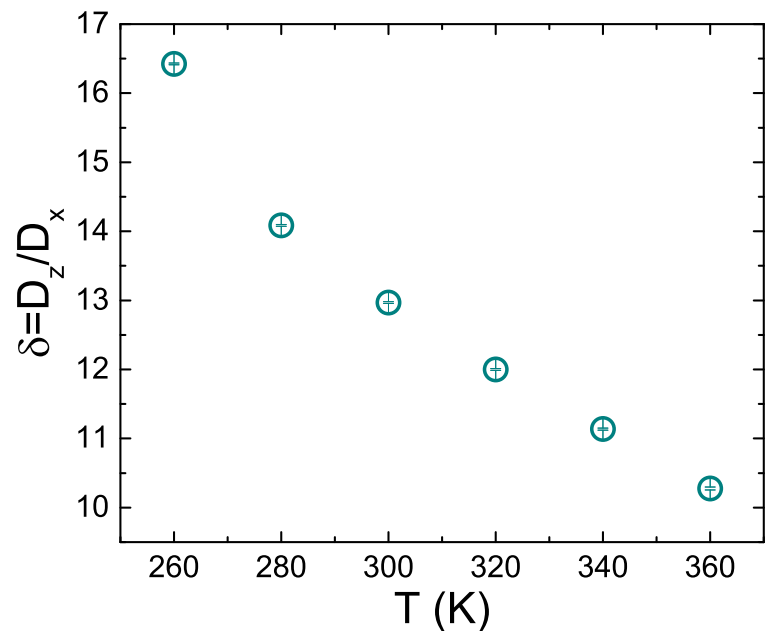

FIG. 9: Degree of anisotropy $\delta=D_{z} / D_{x}$. This quantity tells us how far the ClA molecule is from free rotation around its principal axes.

\section{CONCLUSIONS}

Disorder-disorder phase transition, as those occurring between two liquids, are hard to study due to the concomitant appearance of diffusive translational and rotational degrees of freedom. In a plastic phase, such as that of ClA, the center of mass of the molecules are fixed in a crystalline network so that any change in the structure must be of a purely orientational nature. We have investigated the differences in the microscopic dynamics of the high and low density plastic phases of ClA. We observe that the changes are associated with an anisotropic change of the first neighbor intermolecular interactions, without a change in the crystalline structure. We find, thus, that contrary to the findings of Bée et al. [34], there is a correlation between uniaxial rotation and the overall reorientation motions, so that both movements cannot be considered decoupled as in that work. Finally, we have found that it is possible to observe a change between a high and low density disordered phase with a continuous change in all physical parameters, thus challenging the common believe that such changes must go along a first-order phase transition.

This work was supported by the Spanish Ministry of Science and Innovation through the project FIS201454734-P and by the Generalitat de Catalunya under the project 2014 SGR-581. The UK Science and Technology Facilities Council is gratefully acknowledged for partial financial support and beam time access at the ISIS Facility (RB1220294).
[1] P. Negrier, M. Barrio, J. Ll. Tamarit, L. C. Pardo, and D. Mondieig, Cryst. Growth Des., 12 (3), 15131519 (2012).

[2] P. Negrier, J. Ll. Tamarit, M. Barrio, and D. Mondieig, Cryst. Growth Des., 13 (2), 782791 (2013).

[3] M. Romanini, P. Negrier, J. Ll. Tamarit, S. Capaccioli, M. Barrio, L. C. Pardo, and D. Mondieig, Phys. Rev. B, 85, 134201 (2012).

[4] M. J. Zuriaga, S. C. Perez, L. C. Pardo, and J. Ll. Tamarit, J. Chem. Phys., 137, 054506 (2012).

[5] G. Franzese, G. Malescio, A. Skibinsky, S. V. Buldyrev and H. E. Stanley, Nature, 409, (2001).

[6] T. Speck, C. P. Royall and S. R. Williams, arXiv:1409.0751v2 [cond-mat.stat-mech] (2014).

[7] R. Kurita and H. Tanaka, Science, 306, (2004).

[8] H. Tanaka, R. Kurita and H. Mataki, Phys. Rev. Lett., 92, 025701 (2004).

[9] N. Giovambattista, T. Loerting, B. R. Lukanov and F. W. Starr, Sci. Rep., 390 (2012).

[10] Y. Peng, F. Wang, Z. Wang, A. M. Alsayed, Z. Zhang, A. G. Yodh and Y. Han, Nat. Mat., 14 (2015).

[11] K. J. Chang and M. L. Cohen, Phys. Rev. B, 31 (1985).

[12] O. Mishima and H. E. Stanley Nature 396, 329 (1998).

[13] M. Rovira-Esteva, A. Murugan, L. C. Pardo, S. Busch, M. D. Ruiz-Martín, M. S. Appavou, J. Ll. Tamarit, C. Smuda, T. Unruh, F. J. Bermejo, G. J. Cuello, and S. J. Rzoska, Phys. Rev. B, 81 (9), 092202 (2010).

[14] M. Rovira-Esteva, N. Arul Murugan, L. C. Pardo, S. Busch, J. Ll. Tamarit, G. J. Cuello, F. J. Bermejo, J. Chem. Phys. 136, 124514 (2012).

[15] Y. Katayama, T. Mizutani, W. Utsumi, O. Shimomura, M. Yamakata, and Ken-ichi Funakoshi Nature, 403, 170 (2000).

[16] S. Sastry, and C. A. Angell Nat. Mat. 2, 739 (2005).

[17] K. Kobashi, T. Kyomen, and M. Oguni, J. Phys. Chem. Solids 59, 667 (1998).

[18] J. P. Amoureux, M. Sahour, C. Fernandez, and P. Bodart, Phys. Status Solid, 143, 441 (1994).

[19] T. Clark, T. Mc, O. Knox, H. Mackle, and M. A. McKervey, J. Chem. Soc., Faraday Trans. 73, 1224 (1977).

[20] M. Foulon, T. Belgrand, C. Gors, and M. More, Acta Cryst. B45, 404-411 (1989).

[21] R. Brand, P. Lunkenheimer, and A. Loidl, J. Chem. Phys. 116 (23), 10386 (2002).

[22] L. Carpentier, R. Decressain, and M. Descamps, J. Chem. Phys. 128, 024702 (2008).

[23] B. Ben Hassine, Ph. Negrier, M. Romanini, M. Barrio, R. Macovez, A. Kallel, D. Mondieig and J. Ll. Tamarit, Phys. Chem. Chem. Phys., 18, 10924 (2016).

[24] J. C. Martinez-Garcia, J. Ll. Tamarit, S. Capaccioli, M. Barrio, N. Veglio, and L. C. Pardo, J. Chem. Phys., 132, 164516 (2010).

[25] Y. Huang, D. F. R. Gilson, I. S. Butler, and F. Morin, J. Phys. Chem., 95, 2151-2156 (1991).

[26] Y. Huang, R. M. Paroli, D. F. R. Gilson and I. S. Butler, Can. J. Chem., 71, (1993).

[27] B. Ben Hassine, P. Negrier, M. Barrio, D. Mondieig, S. Massip and J. Ll. Tamarit, Cryst. Growth Des., 15, 41494155 (2015).

[28] F. Affouard, J. Non-Cryst. Solids, 307310, 9 (2002).

[29] P. Negrier, M. Barrio, J. Ll. Tamarit and D. Mondieig, J. Phys. Chem. B, 118, 9595-9603 (2014). 
[30] J. F. Willart and M. Descamps, J. Chem. Phys., 112, 24-22 (2000).

[31] R. Angelini, T. Scopigno, A. Beraud, G. Ruocco, J. NonCrystalline Solids, 352, 4552 (2006).

[32] L.A. Fraczyk, Y. Huang, Spectrochimica Acta Part A, 57, 1061 (2001).

[33] M. Bée and J. P. Amoureux, Mol. Phys., 47 3, 533 (1982).

[34] M. Bée and J. P. Amoureux, Mol. Phys., 48 1, 63 (1983).

[35] F. Affouard and M. Descamps, Phys. Rev. B, 59, 14 (1999).

[36] F. Affouard, E. Cochin, R. Decressain, M. Descamps, Europhys. Lett. 53 (5) 611-617, (2001).

[37] G. Hinze,G. Diezemann, and Th. Basche, Phys Rev. Lett., 93, 20 (2004).

[38] https://www.ill.eu/de/instrumentssupport/instruments-groups/yellowbook/.

[39] http://www.isis.stfc.ac.uk/instruments/iris

[40] V. García Sakai, M. A. Adams, W. S. Howells, M. T. F. Telling, F. Demmel and F. Fernandez-Alonso, Rutherford Appleton Laboratory Technical Report RALTR-2011-004 (Didcot, UK, 2011). Available online at: https://epubs.stfc.ac.uk/work/54579

[41] The latest version of the MANTID software package can be downloaded free of charge from dx.doi.org/10.5286/SOFTWARE/MANTID

[42] S. Mukhopadhyay, Rutherford Appleton Laboratory Technical Report RAL-TR-2014-005 (Didcot, UK, 2014). Available online at: epubs.stfc.ac.uk/work/12135206

[43] P. Bordat, A. Lerbret, M. Descamps, F. Affouard, Mol Simulat, 32, 12-13 (2006).

[44] J. Rodríguez-Carvajal, Recent Developments of the Program FULLPROF, in Commission on Powder Diffraction (IUCr). Newsletter, 26, 12-19 (2001). The software is downloadable at http://www.ill.eu/sites/fullprof/.

[45] T. Roisnel, J. Rodríguez-Carvajal, WinPLOTR: a Windows tool for powder diffraction patterns analysis, Materials Science Forum, Proceedings of the Seventh European Powder Diffraction Conference (EPDIC 7), 118-123, Delhez, R.; Mittenmeijer, E. J. Eds. (2000).

[46] L. C. Pardo et al. arXiv:0907.3711v3 [physics.data-an], (2009).

[47] L. C. Pardo, M. Rovira-Esteva, S. Busch, J.-F. Moulin and J. L. Tamarit, Phys. Rev. E: Stat., Nonlinear, Soft Matter Phys., 84, 046711 (2011).

[48] L. C. Pardo, M. Rovira-Esteva, S. Busch, M. D. RuizMartín and J. L. Tamarit, J. Phys.: Conf. Ser., 325, 012006 (2011).

[49] M. Rovira-Esteva, N. A. Murugan, L. C. Pardo, S. Busch, J. Ll. Tamarit, Sz. Pothoczki, G. J. Cuello, and F. J. Bermejo, Phys. Rev. B, 84, 064202 (2011).

[50] A. Henao, M. Rovira-Esteva, A. Vispa, J. L. Tamarit, E. Guardia and L. C. Pardo, J. Phys.: Condens. Matter, 25, 454217 (2013).

[51] D. Monserrat, A. Vispa, L. C. Pardo, R. Tolchenov, S. Mukhopadhyay and F. Fernandez-Alonso J. Phys.: Conf. Ser., 663, 012009 (2015).

[52] A. Vispa, S. Busch, J. L. Tamarit, T. Unruh, F. Fernandez-Alonso and L. C. Pardo, Phys. Chem. Chem. Phys., 18, 3975 (2016).

[53] S. Busch, C. Smuda, L. C. Pardo, and T. Unruh, J. Am. Chem. Soc., 132 (10), 3232 (2010).

[54] D. S. Sivia and C. J. Carlile, J. Chem. Phys., 96, 170 (1992).

[55] D. S. Sivia and J. Skilling, Data Analysis: A Bayesian
Tutorial, Oxford University Press, (2006).

[56] D. J. C. MacKay, Information Theory, Inference, and Learning Algorithms, Cambridge University Press, (2005).

[57] W. H. Jeffrerys and J. O. Berger, Am. Sci., 80, 64 (1991).

[58] W. Press, H. Grimm, A. Holler, Acta Cryst., Sect. A 35, 881 (1979).

[59] F.J.Bermejo, F.Fernandez-Alonso and C.Cabrillo, Cond. Matt. Phys., 11, 1(53), 95 (2008).

[60] F. Fernandez-Alonso, S. E. McLain, J. W. Taylor, F. J. Bermejo, I. Bustinduy, M. D. Ruiz-Martín and J. F. C. Turner, J. Chem. Phys., 126, 234509 (2016).

[61] F. Fernandez-Alonso, F. J. Bermejo, S. E. McLain, J. F. C. Turner, J. J. Molaison, and K. W. Herwig, Phys. Rev. Lett., 98, 077801 (2007). 